\title{
Fungos associados a grãos de cinco cultivares de café (Coffea arabica $\mathbf{L}$.
}

\author{
Liliana Auxiliadora Avelar Pereira Pasin ${ }^{1,4}$, Júlio Raposo de Almeida ${ }^{2}$ e Mário Sobral de Abreu
}

Recebido em 08/11/2007. Aceito em 14/04/2009

RESUMO - (Fungos associados a grãos de cinco cultivares de café (Coffea arabica L.)). O objetivo deste trabalho foi verificar a micota associada interna e externamente a grãos de café nas cultivares: Catuaí Amarelo, Mundo Novo, Acaiá, Rubi e Icatú, e detectar a ocorrência de Ocratoxina A nestas amostras. Frutos de café foram colhidos na Fazenda Experimental da EPAMIG, situada no município de Lavras/MG. O procedimento para análise de fungos filamentosos foi o método Blotter test. A determinação da ocratoxina A foi realizada por CLAE. Fusarium semitectum foi identificado apenas na cultivar Icatu e F. equiseti foi detectado em todas as cultivares, exceto na Mundo Novo. Três espécies de Penicilium foram isoladas dos grãos: $P$. rugulosum na cultivar Rubi, P. funiculosum na cultivar Icatu e P. variabile nas demais. A cultivar Acaiá apresentou maior incidência de $P$. variabile, seguida por Mundo Novo e Catuaí. A ocorrência de A. niger e A. ochraceus não diferiu entre as cultivares. A presença de fungos na superfície dos grãos foi significativamente superior ao encontrado internamente. Em nenhuma amostra foi detectada a ocorrência de ocratoxina A.

Palavras-chave: Qualidade do café, fungos micotoxigênicos, ocratoxina

ABSTRACT - (Fungi associated with coffee beans (Coffea arabica L.) on five cultivars). The aim of this work was to verify the mycobiota associated both internally and externally with coffee beans on the cultivars "Catuai Amarelo", "Mundo Novo", "Acaiá," "Rubi" and "Icatú" and detect the occurrence of ochratoxin A in these samples. Coffee fruits were harvested on the EPAMIG experiment farm, situated in Lavras /Minas Gerais. The procedure for fungi analysis was the Blotter test. Determination of ochratoxin A was accomplished by HPLC. Fusarium semitectum was identified only on the Icatu cultivar and $F$. equiseti was identified on all cultivars except Mundo Novo. Three species of Penicillium were isolated from the beans: P. rugulosum on the Rubi cultivar, P. funiculosum on the Icatu cultivar and $P$. variabile on the others. The Acaiá cultivar had the highest incidence of $P$. variabile followed by Mundo Novo and Catuaí. The occurrence of $A$. niger and $A$. ochraceus did not differ among the cultivars. The presence of fungi on the surface was significantly higher than inside the beans. None of the samples presented ochratoxin A.

Key words: Coffee quality, fungi micotoxigenic, ochratoxin

\section{Introdução}

A cafeicultura, para o Brasil, possibilita a geração de empregos e arrecadações de impostos importantes para a economia nacional. Por outro lado, para continuidade de lavouras cafeeiras é necessário produzir, e produzir bem, o que significa aumentar a produtividade e melhorar a qualidade.

A qualidade do café é determinada comercialmente por características físicas dos grãos e organolépticas da bebida. $\mathrm{O}$ desenvolvimento de infecções microbianas nos grãos de café pode comprometer tanto o seu aspecto visual quanto o gosto e o aroma. Entre os microorganismos associados a frutos e grãos de café, os fungos filamentosos representam o grupo que pode causar maior dano (Carvalho 1997).

Diversas pesquisas têm procurado assegurar que a qualidade do café não se perca em nenhuma das etapas de produção e processamento dos grãos, levando a estudos de melhoramento genético que propiciem maior vigor das plantas, associado à máxima produtividade (Illy \& Viani 1995; Carvalho 1997; Carvalho et al. 1997). Várias cultivares de café, com importantes características produtivas e vegetativas têm sido desenvolvidas, mas é necessário avaliar também a micota associada aos grãos, em diferentes cultivares, objetivando garantir melhor qualidade do café.

Além da depreciação qualitativa os fungos associados aos grãos de café podem produzir micotoxinas, substâncias tóxicas altamente nocivas à saúde humana (Batista et al. 2003). Vários gêneros de fungos se associam aos frutos do cafeeiro, incluindo os principais produtores de micotoxinas como Aspergillus, Fusarium e Penicillium (Batista et al.
2001). Dentre as micotoxinas presentes em grãos de café, a ocratoxina A é a de maior relevância e representa uma ameaça mais imediata ao consumo e comércio do produto, em função da imposição de limites aos níveis de ocorrência desta micotoxina (Pimenta \& Vilela 2003).

Nesse sentido, o presente estudo teve como objetivo verificar a presença de fungos associados interna e externamente a grãos de café e detectar a ocorrência de ocratoxina A em amostras de diferentes cultivares.

\section{Material e métodos}

Avaliou-se cinco cultivares de café (Acaiá, Mundo Novo, Icatu, Catuaí e Rubi), da espécie Coffea arabica L., em área de produção de sementes da Fazenda Experimental da EPAMIG, localizadas no município de Lavras, MG (Tab. 1). As condições de cultivo foram as recomendadas para a cultura do cafeeiro na região, e todas as plantas foram mantidas sob as mesmas condições de manejo.

Tabela 1. Cultivares e linhagens de Café (Coffea arabica L.) provenientes da Fazenda Experimental da EPAMIG, no município de Lavras-MG.

\begin{tabular}{ccc}
\hline Cultivar & Espaçamento de Plantio & $\begin{array}{c}\text { Ano de Instalação } \\
\text { da lavoura }\end{array}$ \\
\hline Acaiá Cerrado & $3,0 \times 0,7$ & 1996 \\
Mundo Novo & $4,0 \times 0,9$ & 1993 \\
Icatú Amarelo & $3,8 \times 0,8$ & 1993 \\
Catuaí Amarelo & $4,0 \times 0,8$ & 1993 \\
Rubi & $3,0 \times 0,8$ & 1995 \\
\hline
\end{tabular}

Todas as cultivares receberam pulverização com fungicida sistêmico do grupo químico triazóis, para o controle da ferrugem. Realizou-se a colheita quando $80 \%$ dos grãos atingiram o estádio de maturação cereja, sendo colhidos 30 quilos de café, de cada cultivar, que constituiram as amostras analisadas.

\footnotetext{
1 Universidade do Vale do Paraíba, Centro de Estudos da Natureza/ Setor de Botânica

2 Universidade de Taubaté, Departamento de Ciências Agrárias, Taubaté, SP, Brasil

3 Universidade Federal de Lavras, Departamento de Fitopatologia, Lavras, MG, Brasil

4 Autor para correspondência: Ipasin@univap.br
} 
O processo de secagem dos frutos foi realizado em terreiro de concreto, imediatamente após a colheita. Os grãos permaneceram no terreiro até atingirem o teor de umidade de aproximadamente $12 \%$, aos 20 dias, e posteriormente foram beneficiados, acondicionados em sacos de papel duplo e armazenados no laboratório de Controle de Enfermidades de Plantas do Departamento de Fitopatologia da UFLA, até a realização das análises, realizadas 15 dias após a colheita.

Das amostras beneficiadas de cada cultivar retirou-se aleatoriamente 100 grãos, para posterior distribuição em placas de Petri em condições assépticas, conforme a técnica Blotter Test (Tempe, 1963). Cada placa com 25 grãos foi considerada uma repetição. Após plaqueamento, os grãos foram incubados a $20^{\circ} \mathrm{C} \pm 2{ }^{\circ} \mathrm{C}$ em câmara com fotoperíodo de 12 horas durante oito dias. A micota associada aos grãos foi avaliada pelo exame de cada grão, individualmente, em microscópio estereoscópio. A identificação das espécies foi realizada pela análise das estruturas morfológicas dos fungos em microscópio de luz.

Para análise dos fungos associados aos grãos, utilizou-se delineamento experimental em blocos casualisados, constando de quatro blocos, em esquema fatorial $5 \mathrm{X} 2$, considerando as cinco cultivares e a desinfestação superficial de metade dos grãos com hipoclorito de sódio a $1 \%$.

$\mathrm{Na}$ análise estatística utilizou-se o procedimento GLM do sistema estatístico SAS ${ }^{(}$(Sas Institute, 1992). Os dados foram transformados em $\sqrt{ } x+0,5$ para homogeneidade e sumetidos a análise de variância e as médias comparadas pelo teste de Tukey $(\mathrm{P} \leq 0.05)$.

Para detecção da Ocratoxina A, foram coletados aleatoriamente na lavoura, em diversos pontos, $30 \mathrm{Kg}$ de frutos de cada cultivar. Posteriormente, retirou-se $1 \mathrm{Kg}$ de cada amostra de café verde beneficiado, constituindo as sub-amostras, que depois de trituradas foram devidamente homogeneizadas para a retirada de $50 \mathrm{~g}$ para a realização da análise.

As análises de Ocratoxina A foram realizadas por Cromatografia Liquida de Alta Eficiência (CLAE) no Laboratório de Controle de Qualidade e Segurança Alimentar/LACQSA em Belo Horizonte. A metodologia fundamenta-se na extração da ocratoxina A com solução de metanol: bicarbonato de sódio $3 \%(1: 1, \mathrm{v} / \mathrm{v})$ purificação de extrato com coluna de imunoafinidade (Ochratest-Vicam) separação e quantificação da ocratoxina A em cromatógrafo líquido em coluna Shimpack C18 CLC ODS (M) $250 \times 4,6 \mathrm{~mm}$, como fase móvel acetonitrila:metanol:água:ácido acético (35:35:29:1,v/v/v/v) com fluxo de $0,8 \mathrm{~mL} / \mathrm{min}$, detector de fluorescência com excitação em $332 \mathrm{~nm}$ e emissão em $476 \mathrm{~nm}$. O limite de detecção do método foi de $0,12 \mu \mathrm{g} / \mathrm{Kg}$ e o limite de quantificação é de $0,20 \mu \mathrm{g} / \mathrm{Kg}$. Para a análise foram utilizadas triplicatas das amostras.

\section{Resultados e discussão}

Foram detectadas nos grãos das diversas cultivares de café, oito espécies de fungos dos gêneros Aspergillus, $\mathrm{Fu}$ sarium, Penicillium e Cladosporium (Tab.2). Verificou-se a ocorrência de Fusarium semitectum Berk \& Ravenel apenas na cultivar Icatu. Fusarium equiseti (Corda) Sacc. não foi detectado na cultivar Mundo Novo, entretanto verificou- se alta incidência deste fungo na parte externa dos grãos das demais cultivares. Trabalhando com amostras de café beneficiado de diversos municípios da região Sul de Minas Gerais, Freitas (2000) constatou que as lavouras de Catuaí e Icatu apresentaram índices de infestação por Fusarium sp. inferiores aos apresentados nas lavouras formadas por outras cultivares, sugerindo que a incidência fúngica ocorre de maneira diferenciada nas várias cultivares.

Não se observou sintoma associado ao Fusarium em nenhuma das cultivares avaliadas embora o fungo estivesse presente nas amostras de café. Como não foram detectados sintomas de fusariose nas plantas das quais foram coletadas as amostras, supõe-se que os frutos tenham sido infectados por conídios recém-germinados do fungo, disseminados pelo vento, chuva ou insetos. A ocorrência de $F$. semitectum apenas na cultivar Icatu pode ser explicada pela contaminação casual, ainda no campo, por conídios deste fungo, e não por alguma característica inerente a esta cultivar.

A associação de espécies de Penicillium em grãos café beneficiado foi evidenciada por Batista \& Chalfoun (2007), que identificaram $P$. brevicompactum Dierckx, $P$. citrinum Thom, $P$. commune Thom, $P$. minioluteum Dierckx, $P$. variabile Sopp, $P$. expansum Link e P. corylophilum Dierckx, associadas a grãos de café coletados em 11 municípios da região Sul de Minas Gerais, no entanto, neste estudo, apenas o Penicillium variabile foi detectado nas amostras obtidas das cinco cultivares analisadas. Freitas (2000) observou que os valores médios de ocorrência de espécies de Penicillium foram mais elevados em lavouras de Catuaí e Icatu. Neste trabalho, a cultivar Icatu foi a única em que se verificou a ocorrência de Penicillium funiculosum Thom. A cultivar Catuaí apresentou menor incidência de $P$. variabile, diferindo estatisticamente $(\mathrm{P} \leq 0,05)$ do encontrado nas cultivares Acaiá e Mundo Novo. Este fungo não foi detectado nas demais cultivares (Tab.2). Penicillium rugulosum Thom. foi detectado apenas na cultivar Rubi.

Espécies de Penicillium e Aspergillus são adaptadas a ambientes com baixa umidade e podem crescer em qualquer matéria orgânica que contenha grau de umidade em equilíbrio com a umidade do ambiente entre 65 e $90 \%$. De ocorrência cosmopolita, estão entre os mais bem sucedidos

Tabela 2. Porcentagem de incidência fúngica em grãos de café (Coffea arabica L.) processados por via seca, em diferentes cultivares.

\begin{tabular}{|c|c|c|c|c|c|c|c|c|c|c|c|c|c|c|c|c|}
\hline \multirow[t]{2}{*}{ Cultivares } & \multicolumn{2}{|c|}{ F. semitectum } & \multicolumn{2}{|c|}{ F. equiseti } & \multicolumn{2}{|c|}{ P. rugulosum } & \multicolumn{2}{|c|}{ P. variable } & \multicolumn{2}{|c|}{ P. funiculosum } & \multicolumn{2}{|c|}{ A. ochraceus } & \multicolumn{2}{|c|}{ A. niger } & \multicolumn{2}{|c|}{ Cladosporium } \\
\hline & ND & D & ND & D & ND & D & ND & D & ND & D & ND & D & ND & D & ND & D \\
\hline Acaiá & $00 \mathrm{Aa}$ & $00 \mathrm{Aa}$ & $57 \mathrm{Ab} \mathrm{C}$ & $4 \mathrm{Ba}$ & $00 \mathrm{Aa}$ & $00 \mathrm{Aa}$ & $95 \mathrm{Aa}$ & $03 \mathrm{Ba}$ & $00 \mathrm{Aa}$ & $00 \mathrm{Aa}$ & $18 \mathrm{Aa}$ & $03 \mathrm{Ba}$ & $02 \mathrm{Aa}$ & $1 \mathrm{Aa}$ & $87 \mathrm{Aa}$ & $02 \mathrm{Ba}$ \\
\hline $\mathrm{MN}$ & $00 \mathrm{Aa}$ & $00 \mathrm{Aa}$ & $00 \mathrm{AaC}$ & $0 \mathrm{Aa}$ & $00 \mathrm{Aa}$ & $00 \mathrm{Aa}$ & $72 \mathrm{Ab}$ & $02 \mathrm{Ba}$ & $00 \mathrm{Aa}$ & $00 \mathrm{Aa}$ & $09 \mathrm{Aa}$ & $01 \mathrm{Aa}$ & $04 \mathrm{Aa}$ & $0 \mathrm{Aa}$ & $92 \mathrm{Aa}$ & $02 \mathrm{Ba}$ \\
\hline Icatu & $83 \mathrm{Ab}$ & $03 \mathrm{Ba}$ & $87 \mathrm{Acc}$ & $2 \mathrm{Ba}$ & $00 \mathrm{Aa}$ & $00 \mathrm{Aa}$ & $00 \mathrm{Ad}$ & $00 \mathrm{Ba}$ & $79 \mathrm{Ab}$ & $04 \mathrm{Ba}$ & $08 \mathrm{Aa}$ & $02 \mathrm{Aa}$ & $02 \mathrm{Aa}$ & $00 \mathrm{Aa}$ & $92 \mathrm{Aa}$ & $04 \mathrm{Ba}$ \\
\hline Catuaí & $00 \mathrm{Aa}$ & $00 \mathrm{Aa}$ & $77 \mathrm{Acc}$ & $3 \mathrm{Ba}$ & $00 \mathrm{Aa}$ & $00 \mathrm{Aa}$ & $37 \mathrm{Ac}$ & $04 \mathrm{Ba}$ & $00 \mathrm{Aa}$ & $00 \mathrm{Aa}$ & $12 \mathrm{Aa}$ & $02 \mathrm{Aa}$ & $12 \mathrm{Ab}$ & $01 \mathrm{Aa}$ & $86 \mathrm{Aa}$ & $03 \mathrm{Ba}$ \\
\hline Rubi & $00 \mathrm{Aa}$ & $00 \mathrm{Aa}$ & $74 \mathrm{AcC}$ & $4 \mathrm{Ba}$ & $82 \mathrm{Aa}$ & $04 \mathrm{Ba}$ & $00 \mathrm{Ad}$ & $00 \mathrm{Aa}$ & $00 \mathrm{Aa}$ & $00 \mathrm{Aa}$ & $16 \mathrm{Aa}$ & $04 \mathrm{Aa}$ & $02 \mathrm{Aa}$ & $00 \mathrm{Aa}$ & $87 \mathrm{Aa}$ & $01 \mathrm{Ba}$ \\
\hline
\end{tabular}

Médias seguidas pela mesma letra maiúscula nas linhas, e minúsculas nas colunas, para cada fungo, são estatisticamente iguais pelo teste de Tukey (P $\leq 0,05)$.

D - grãos desinfestados com $\mathrm{NaOCl}$ a 1\%; ND - grãos não desinfestados 
e abundantes organismos vivos, sendo normalmente associados a grãos armazenados ou danificados (Chalfoun \& Batista, 2003).

Aspergillus niger Tiegh. e Aspergillus ochraceus G. Wilh. são descritos em vários relatos associados ao café (Carvalho et al. 1997; Pimenta \& Vilela 2003; Batista \& Chalfoun 2007). Este trabalho confirma a ocorrência destes fungos em grãos de café de diferentes cultivares e no caso não houve diferença significativa $(\mathrm{P} \leq 0.05)$ na incidência.

A micobiota externa aos grãos é significativamente maior que a interna; no entanto, apesar de reduzida porcentagem média de ocorrência, constatou-se a ocorrência interna de todos os fungos detectados, nas diferentes cultivares.

A colonização interna pelos fungos pode ser explicada por danos causados por insetos, ou mesmo fungos fitopatogênicos, ácaros ou condições climáticas adversas. Outra explicação é a ocorrência da ruptura de estruturas da parede celular por alterações nas pectinas, celulose, hemicelulose e ligninas nos frutos. Estes compostos conferem uma estrutura mais rígida aos frutos de café, e a degradação natural torna os frutos mais suscetíveis à ocorrência fúngica (Batista \& Chalfoun 2007).

O limite máximo de ocratoxina A para cereais $(5,0 \mu \mathrm{g} /$ $\mathrm{Kg})$ e seus subprodutos $(3,0 \mu \mathrm{g} / \mathrm{Kg})$ tem sido estabelecido pela Comissão de Regulamentação da União Européia EC n.472 (European Community 2002), que também estabelece um limite de $5 \mu \mathrm{g} / \mathrm{Kg}$ para grãos de café torrado e moído. Portanto, nas condições deste estudo, os grãos de todas as cultivares avaliadas têm um excelente padrão, pois não se detectou a micotoxina.

Dos fungos detectados apenas A. ochraceus e A. niger são citados como produtores de ocratoxina A (Batista et al. 2003). Apesar de $A$.ochraceous ter ocorrido externamente em $18 \%$ das amostras da cultivar Acaiá, não se detectou a ocorrência de ocratoxina A nessas cultivares, sugerindo que a associação de espécies toxigênicas externamente a grãos não implica na síntese deste metabólito. Os resultados confirmam que a ocorrência desta toxina é dependente de vários fatores (Chalfoun et al. 2000), entre os quais a composição química dos grãos, já que certas substâncias, como a cafeína, podem inibir substancialmente a produção de micotoxinas. A cafeína (1,3,7 trimetilxantina) constitui componente natural em café, com atividade fungistática e efeito antimicotoxigênico simultâneo (Buchanan et al. 1983). Este composto pode atuar como agente protetor contra a produção de aflatoxina B1 em grãos de café, mesmo em condições climáticas favoráveis ao crescimento do fungo produtor (Micco et al. 1992). O mesmo pode ter ocorrido para ocratoxina A nas condições deste estudo.

Fujii et al. (2004) também evidenciaram que a cafeína pode exercer efeito fungistático em grãos de café infectados por vários gêneros fúngicos. Os autores sugerem que a ação inibitória no crescimento micelial decorre da similaridade estrutural entre purinas e cafeína, que consistem de compostos cíclicos nitrogenados, cuja inibição competitiva reduziria a síntese de ácidos nucléicos, afetando conseqüentemente o desenvolvimento fúngico. Chalfoun et al. (2000) relataram que a cafeína pode exercer atividade biológica contra vários fungos, inclusive os toxigênicos. Neste trabalho, a micobiota interna foi acentuadamente inferior à externa do grão, sugerindo que a cafeína pode ter exercido efeito inibidor no desenvolvimento destes fungos.

Levantamentos realizados em amostras de café para detecção da ocorrência de micotoxinas demonstraram que a freqüência é reduzida e, quando a toxina é encontrada, o nível é baixo (Tsubouchi et al.1987; Levi 1990; Mantle \& Chow 2000; Pimenta \&Vilela 2003), o que foi confirmado neste trabalho. No entanto, também há registros de elevada presença de fungos internamente em amostras de grãos de café, sendo também detectado a ocratoxina A em níveis superiores aos propostos pela União Européia (Truckess et al. 1999). A alta porcentagem de ocorrência de fungos toxigênicos sugere que outros fatores, além da composição do substrato, podem influir na sua ocorrência e consequente produção de toxinas.

Dentre os fatores que favorecem o desenvolvimento fúngico ressaltam-se temperatura, predomínio de linhagens toxigênicas, competição por substrato e cultivares resistentes (Scussel, 1998). Esse autor ressalta que variedades de grãos com pericarpo mais resistente apresentam maior resistência à penetração e proliferação de fungos e, consequentemente, a produção de micotoxinas é dificultada ou suprimida. Como a ocorrência interna dos fungos associados aos grãos foi reduzida em todas as cultivares avaliadas, pode-se inferir que estas podem constituir excelentes alternativas quando se busca qualidade dos grãos.

\section{Agradecimentos}

Os autores agradecem à CAPES pelas bolsa concedida a L.A.A.P.Pasin, ao CBP\&D-Café, pelo financiamento do projeto, ao Laboratório de Controle de Qualidade de Segurança Alimentar/LACQSA/Belo Horizonte pelo valioso auxilio nas análises de Ocratoxina, à UFLA (Universidade Federal de Lavras) e EPAMIG (Empresa de Pesquisa Agropecuária de Minas Gerais), pela oportunidade de realização do trabalho.

\section{Referências bibliográficas}

Batista, L.R.; Chalfoun, S.M. \& Prado, G. 2001. Identificação de espécies toxigênicas de Aspergillus associadas aos grãos de café armazenados. Revista Brasileira de Armazenamento 3: 11-16. Especial Café.

Batista, L.R.; Chalfoun, S.M.; Prado, G.; Schwan, R.F. \& Wheals, A.E. 2003. Toxigenic fungi associated with processed (green) coffee beans (Coffea arabica L.) International Journal of Food Microbiology 85:293-300.

Batista, L.R. \& Chalfoun, S.M. 2007. Incidência de Ocratoxina A em diferentes frações do café (Coffea arabica L.) Bóia, mistura e varrição após secagem em terreiros de terra, asfalto e cimento. Ciência e Agrotecnologia 31: 804-813.

Buchanan, R.L.; Harry, M.A \& Gealt, M.A. 1983. Caffeine inhibition of steigmatocystin, citrinin, and patulin production. Journal of Food Science 48: 1226-1228.

Carvalho, V.D. 1997. Cafeicultura empresarial: produtividade e qualidade - qualidade do café.

Lavras, UFLA/FAEPE.

Carvalho, V.D.; Chagas, S.J.R. \& Souza, S.M.C. 1997. Fatores que afetam a qualidade do café. Informe Agropecuário 18: 5-20. 
Chalfoun, S. M. \& Batista, L.R. 2003. Fungos associados a frutos de café Aspergillus \& Penicillium. Brasília, Embrapa.

Chalfoun, S. M.; Pereira, M.C. \& Angélico, C.L. 2000. Efeito da cafeína (1,3,7-triemethylxanthina) sobre o crescimento micelial de fungos associados ao café. Revista Brasileira de Armazenamento 1: 5053, Especial.

European Community 2002. Commission regulation (EC) 472/2002, amending regulation (EC) 466/2001 setting maximum level for certain contaminats in food stuffs. Official Journal of the European Communities L75: 18-20

Freitas, R.F. 2000 Fungos associados a grãos de café (Coffea arabica L.) beneficiado de diversos municipios da Região Sul de Minas Gerais. Lavras, UFLA.

Fujii, S.; Assunção, F.G.A.; Taniwaki, M.H.; Scholz, M.B.S.; Gomes, R.J.H.C. \& Hirooka, E.Y. 2004. Atividade fungistática "in vitro" de cafeína em fungos associados com grãos de café. Acta Scientiarum Agronomy 26: 279-285.

Illy, A. \& Viani, R. 1995. Espresso coffee: the chemistry of quality. San Diego, Academic Press.

Levi, C. P. 1990. Mycotoxins in coffee. Journal of Association Official Analytical Chemistry 6: 1282-1285.
Mantle, P. G. \& Chow, A. G. 2000. Ochratoxin formation in Aspergillus ochraceus with particular reference to spoilage of coffee. International Journal of Food Microbiology 56: 105-109.

Micco, M.; Miraglia, M.; Brera, C.; Desiderio, C. \& Masci, V. 1992. The effect of roasting on the fate aflatoxin $\mathrm{B} 1$ in artificially contaminated green coffee beans. Mycotoxin Research 8: 93-97.

Pimenta, C.J. \& Vilela, E.R. 2003. Composição microbiana e Ocratoxina A no Café (Coffea arabica L.) submetidos a diferentes tempos de espera antes da secagem. Ciência e Agrotecnologia 27:1315-1320.

SAS Institute. 1992. SAS technical report SAS/TAT software: changes and enhacenment release 607. Cary Nc., SAS Institute.

Scussel, V. M. 1998. Micotoxinas e Alimentos. Florianópolis, Insular.

Tempe, J. de 1963. The blotter method for seed health testing. Proceedings of the International Testing Association, Copehagen. 28:133-151.

Truckess, M.W.; Giler, J.; Young, K.; White, K.D.; Page, S.W. 1999. Determination and survey of ochratoxin A in wheat, barley, and coffee. Journal of AOAC International. 8: 85-87

Tsubouchi, H.; Yamamoto, K.; Hisada, K.; Sakabe, Y; Udagawa, S. 1987. Effect of roasting on ochratoxin A level in green coffee beans inoculated with Aspergillus ochraceus. Mycopathologia 97: 111-115 